\title{
THERMAL/STRUCTURAL ANALYSIS OF A SCRF PHOTOCATHODE ELECTRON GUN CAVITY*
}

\author{
T. Schultheiss, M. Cole, J. Rathke, \\ Advanced Energy Systems, Medford, NY11763, USA
}

\begin{abstract}
The all niobium superconducting RF photocathode electron gun being developed by Advanced Energy Systems and Brookhaven National Laboratory was analyzed to determine an optimum cavity configuration. An overview of this program appears under a separate title [M. Cole, this conference, ref 1]. Details of the thermal and structural analysis and results for the final configuration are presented in this paper. A $1.8 \mathrm{~K}$ superfluid helium bath was assumed on the outside of the cavity. The assumptions that were used on the superfluid side as well as the thermal and structural properties of RRR 250 niobium are given. Heat loads were developed from a SUPERFISH model and were included for surface temperatures between 1.8 and $8 \mathrm{~K}$. Thermal conductivity, RF heat loads, and Kapitza resistance all being highly non-linear require an iterative thermal solution. Laser power and power density limits were determined and are presented. The final cavity wall thickness provides adequate structural stiffness and a broad enough thermal path to decrease the superfluid side heat flux to acceptable levels.
\end{abstract}

\section{INTRODUCTION}

Linear accelerators using superconducting radio frequency (SRF) cavities are the preferred method when high average beam currents are to be accelerated to high energies and when high-brightness beams are required. SRF linacs can transport the highest average currents, minimize emittance growth, minimize beam spill because of the large inter-cavity apertures that can be used, lead to better phase and amplitude stability because of the high stored energy of the cavities and can support the highest "real-estate" accelerating gradients; thereby leading to the brightest, most efficient and most compact accelerator system for the given application.

In this project we are evaluating integration of the photocathode into the SRF cavity. This will provide a substantial improvement in performance and efficiency as compared to using a normal conducting RF gun or other electron source to inject into a superconducting RF

* Work supported by the U.S. DOE., Office of Basic Energy Sciences, under Contract Nos. DE-AC02-98CH10886DOE, the SBIR grant DE-FG02-99ER82724, and under CRADA No. BNL-C-00-15. Such support does not constitute an endorsement by DOE of the views expressed in this paper. accelerator. Operating costs will be substantially less than for a normal conducting RF gun because of the much lower losses in the SRF gun and the elimination of the need for high-power CW RF sources. The concept avoids mixed technology (superconducting and normal conducting RF) resulting in a simpler design, so that the entire injector will be considerably more compact than existing injectors. Since no foreign material is introduced into the superconducting environment and a complicated mounting structure is not required, the specific means of integration proposed here is an elegant solution as compared to other SRF photocathode gun schemes. The focus of this paper is thermal and structural analysis used to determine the feasibility of this approach.

\section{MODELING}

The superconducting electron gun was analyzed thermally and structurally to determine optimum wall thickness and to simplify the cavity structure. An earlier configuration included a protruding cathode surface, a flat wall, and niobium stiffeners. In the present configuration the cathode surface smoothly integrates with a curved cavity shape that does not require any stiffeners, reducing its complexity, figure 1. To keep the overall helium inventory low the design temperature of the cavity was set

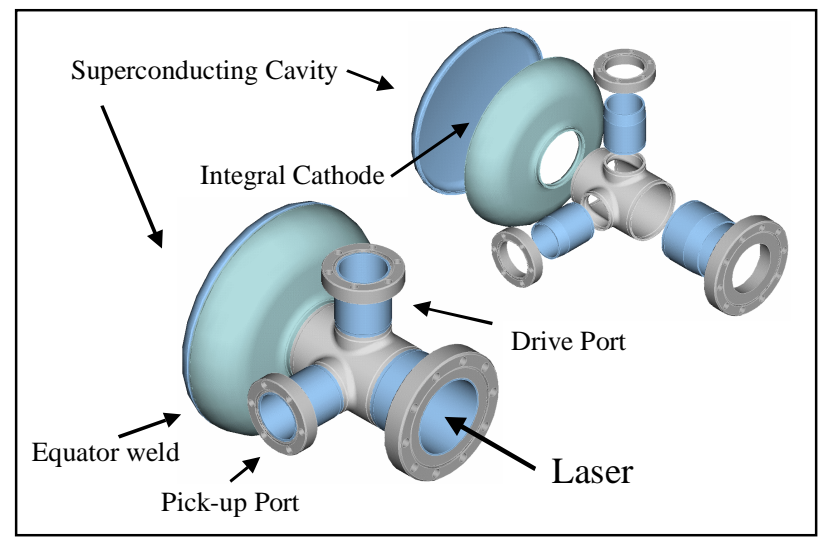

Figure 1, SCRF cavity configuration

to the superfluid temperature range. This reduced the temperature on the RF surfaces which in turn reduced the temperature dependent RF heat loads. SUPERFISH runs were completed for several different surface temperature assumptions and the temperature dependent heat loads were obtained, Table 1. The associated SUPERFISH segments are given in figure 2 where the segments are shown as axisymmetric sectors. Heat loads for segment 
numbers three through nine were taken directly from SUPERFISH output. Heat loads for segment numbers ten through eighteen include the SUPERFISH calculated loads plus the laser load. The laser load is assumed to have a gaussian distribution peaking at the center of segment 10. The effects of the laser load are insignificant in segments 15 through 18, therefore, since these segments were modeled with a single segment in SUPERFISH they all have the same heat load.

Table 1, Segment Heat Loads

\begin{tabular}{|c|c|c|c|c|}
\hline \multirow{2}{*}{ Segment } & \multicolumn{4}{|c|}{ Surface Heat Loads mW/cm } \\
\cline { 2 - 5 } & $1.8 \mathrm{~K}$ & $4.2 \mathrm{~K}$ & $6 \mathrm{~K}$ & $8 \mathrm{~K}$ \\
\hline 3 & 7.40 & 184.2 & 425.6 & 642.4 \\
\hline 4 & 7.28 & 181.4 & 419.0 & 632.3 \\
\hline 5 & 6.94 & 172.9 & 399.4 & 602.7 \\
\hline 6 & 7.05 & 175.6 & 405.6 & 612.2 \\
\hline 7 & 5.85 & 145.7 & 336.5 & 507.9 \\
\hline 8 & 1.98 & 49.3 & 113.9 & 171.9 \\
\hline 9 & .00271 & .675 & 1.56 & 2.35 \\
\hline 10 & 9854. & 9896. & 9953. & 10004. \\
\hline 11 & 6992 & 7034. & 7091. & 7142. \\
\hline 12 & 2046. & 2087. & 2144. & 2196. \\
\hline 13 & 113.5 & 155.3 & 212.3 & 263.5 \\
\hline 14 & 2.091 & 43.87 & 100.9 & 152.1 \\
\hline 15 & 1.747 & 43.53 & 100.6 & 151.8 \\
\hline 16 & 1.747 & 43.53 & 100.6 & 151.8 \\
\hline 17 & 1.747 & 43.53 & 100.6 & 151.8 \\
\hline 18 & 1.747 & 43.53 & 100.6 & 151.8 \\
\hline
\end{tabular}

Thermal conductivity of niobium is also a strong function of temperature and is included in the thermal model. Values used for thermal conductivity are given in Table 2.

Table 2, Material properties of niobium

\begin{tabular}{|c|c|c|}
\hline $\begin{array}{c}\text { Temp } \\
\mathrm{K}\end{array}$ & $\begin{array}{c}\text { Thermal } \\
\text { Conductivity } \\
\text { W/m-K }\end{array}$ & $\begin{array}{c}\text { Yield Strength } \\
\text { ksi }\end{array}$ \\
\hline 2 & 5 & - \\
\hline 4 & 50 & 61.0 \\
\hline 300 & 52 & 9.7 \\
\hline
\end{tabular}

Coolant side thermal boundary conditions were based on a $1.8 \mathrm{~K}$ superfluid temperature $\mathrm{T}_{\mathrm{b}}$ and the heat flux $\Phi_{\mathrm{s}}$ through the surface is determined by the temperature dependent Kapitza conductivity $\mathrm{H}_{\mathrm{k}}$ [2]. This is given by the following equations:

$$
\begin{gathered}
\Phi_{s}=H_{k}\left(T_{b}, \Delta T\right) \Delta T \\
H_{k}\left(T_{b}, \Delta T\right)=.02 T_{b}^{4.65} f\left(T_{b}, \Delta T\right) \\
f\left(T_{b}, \Delta T\right)=1+1.5 t+t^{2}+.25 t^{3} \\
t=\Delta T, \Delta T \leq 1.4 K
\end{gathered}
$$

When $\Delta \mathrm{T}$ is greater than $1.4 \mathrm{~K}$, the surface flux exceeds the critical heat flux for superfluid helium and the local conductivity is greatly reduced. The heat flow will then redistribute if the wall thickness is large enough.

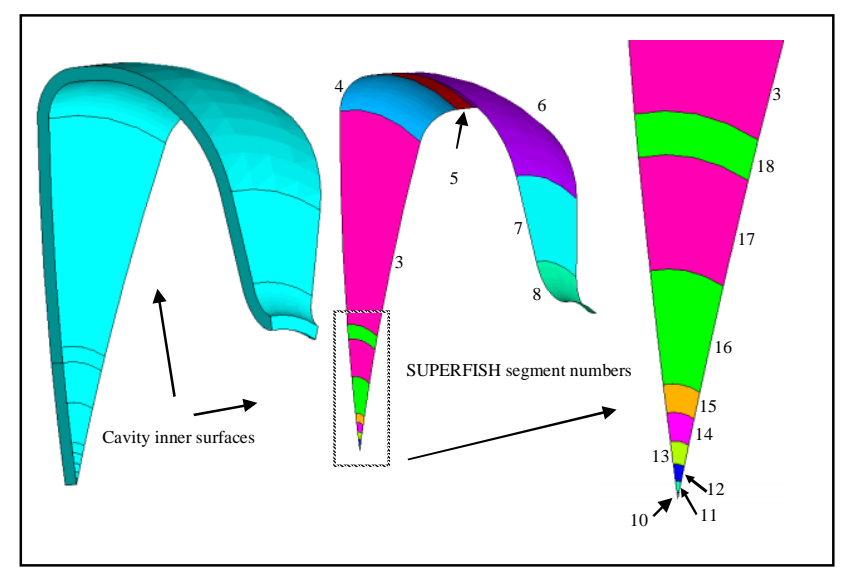

Figure 2, Segment heat loads

\section{THERMAL RESULTS}

An axisymmetric model was sufficient for optimizing the wall thickness. This model and the general boundary conditions are shown in figure 3. The highly non-linear nature of the thermal model made steady state convergence using the normal techniques difficult. By including density and specific heat in a thermal transient the steady state solution was reached in a reasonable number of solution steps. This turned out to be the most effective solution method for the input encountered here.

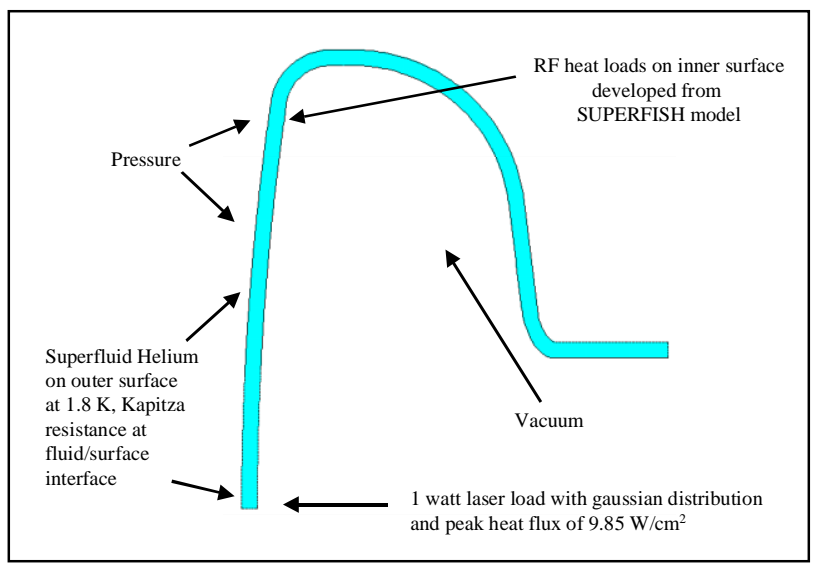

Figure 3, Axisymmetric cavity model

Temperature solutions were determined for several different wall thicknesses to determine the effects. The results of wall thicknesses of $1.5,2.5$, and $3.5 \mathrm{~mm}$ are shown on figure 4 . Limitations of the Kapitza conductivity, about $1 \mathrm{~W} / \mathrm{cm}^{2}$ for a superfluid helium niobium interface, cause a local temperature rise at the center of the laser load. By increasing the wall thickness, the local heat redistributes and dissipates over a larger area, lowering the peak wall temperature. This is shown 


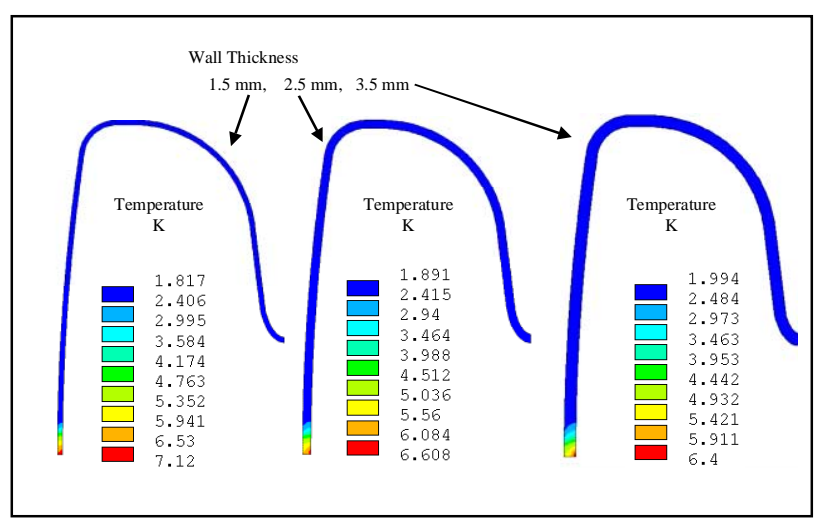

Figure 4, Temperatures for different wall thicknesses

in figure 5 where peak temperature is plotted as a function of wall thickness. The slope of the curve also indicates that the improvement in temperature slowly decreases as the thickness is increased. Going beyond $3.5 \mathrm{~mm}$ in wall thickness does not appear necessary from the thermal results. The next step was to compare the wall thickness to the stress resulting from pressure differential.

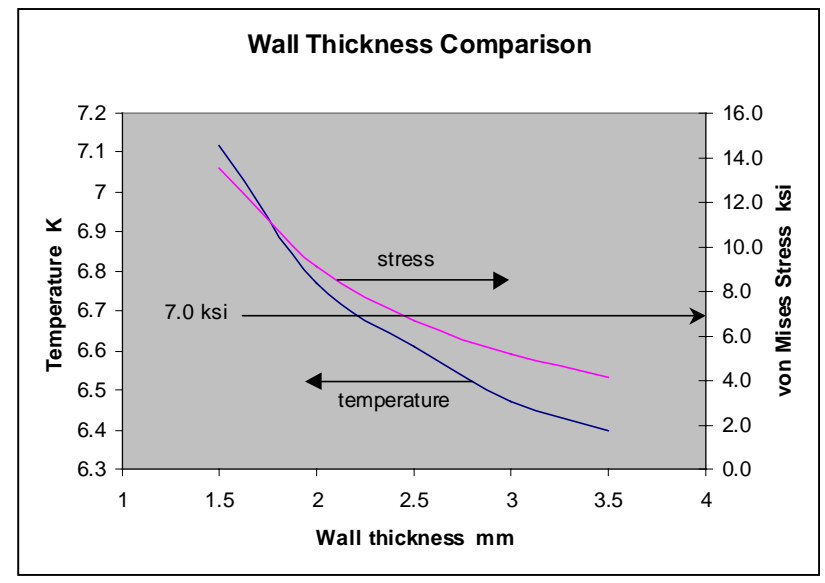

Figure 5, Wall thickness comparison

\section{STRUCTURAL RESULTS}

Structural results include a pressure load of one atmosphere on the outside of the cavity. Figure 6 shows the von Mises stress results for the same thicknesses as the temperature comparison. These stresses were plotted and shown on figure 5 , comparing the stress as a function of wall thickness. The stress decreases through $3.5 \mathrm{~mm}$ wall thickness. Since we did not want to go larger than $3.5 \mathrm{~mm}$ this was the wall thickness chosen.

A manufacturing notch for the equator weld process was added to the structural model to check the integrity of the cavity geometry and the model was run with the prior pressure loads. Figure 7 shows the von Mises, maximum, and minimum principal stress with the notch. The results show that the notch is located away from the peak stress region of the cavity. Furthermore, the notch makes the geometry slightly more flexible resulting in a small decrease in the von Mises stress.

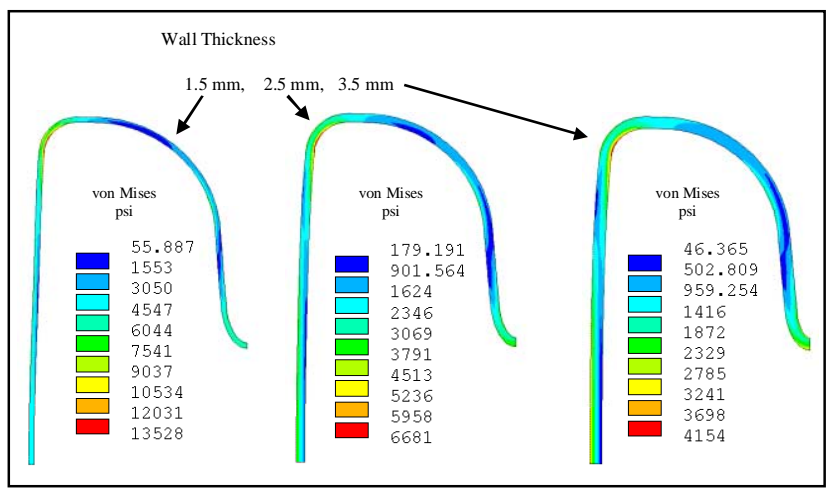

Figure 6, von Mises stress comparison

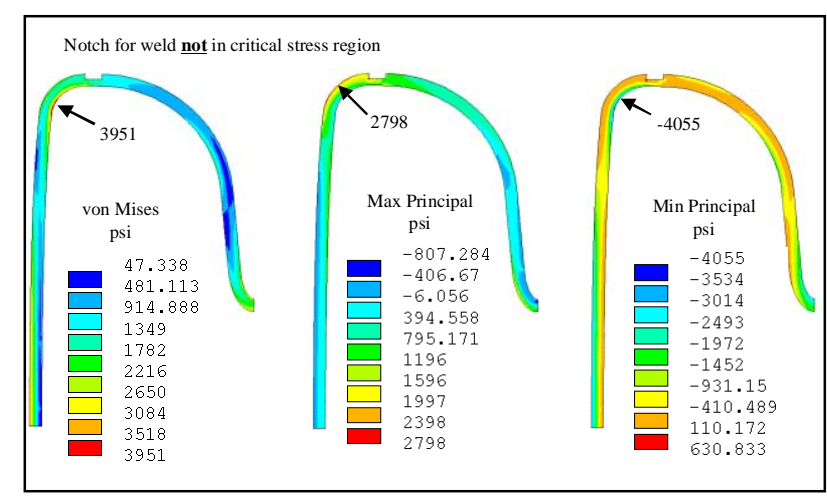

Figure 7, Effects of manufacturing notch

\section{CONCLUSIONS}

Optimization of the superconducting gun wall thickness has been demonstrated as a balance between wall temperature, wall stresses, and wall thickness (which relates to material cost). Thermal analysis has shown that in this case critical heat flux can be overcome by increasing the wall thickness, thereby achieving adequate temperature and stress margins. Additional information about this program can be found in the web listing of [3].

\section{REFERENCES}

[1] M. Cole, et. al., "Progress Toward an All Niobium Superconducting RF Photcathode Electron Gun"; Proceedings of PAC2001, WPAH077, Chicago, ILL., 2001.

[2] H. Padamsee et al., RF Superconductivity for Accelerators, John Wiley and Sons, New York, New York, 1998.

[3] http://www.aesys.net/

[4] J. Knobloch et al., "High-Field Q Slope in Superconducting Cavities Due to Magnetic Field Enhancement at Grain Boundaries"; Proceedings of the 9th Workshop on RF Superconductivity, Santa Fe, NM, 1999 